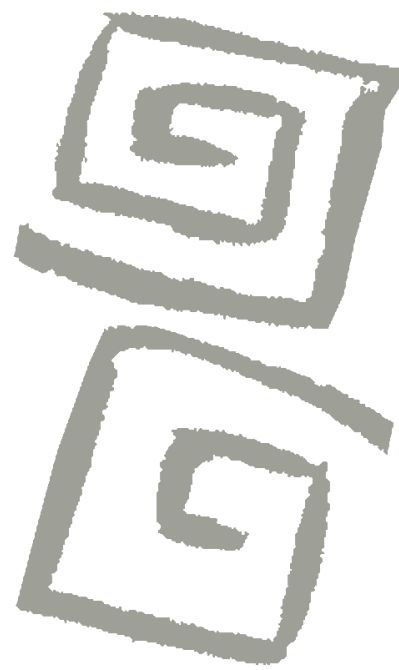

\title{
Aproximación etnográfica al concepto de salud y enfermedad en la tribu turkana: proyecto para la mejora de la salud de los nómadas del Triángulo de Ilemi, Kenia
}

\author{
An ethnographic approach to the concepts of health \\ and disease in the Turkana tribe: a project to improve \\ the health of the nomads of the Ilemi Triangle, Kenya
}

José Javier Cardós García ${ }^{1}$, Manuel Lillo Crespo², Andrés Climent Rubio ${ }^{3}$, Emiliano Hernández Urrutia ${ }^{4}$, Beatriz Mejias Moreno ${ }^{5}$

${ }^{1}$ Graduado en Enfermería Magíster en Urgencias y Emergencias. Enfermero, Clínica Vistahermosa, Alicante, España. $\square$ (iD)

${ }^{2}$ Doctor en Antropología de la Salud y Enfermería. Profesor Asociado, Departamento de Enfermería, Facultad de Ciencias de la Salud, Universidad de Alicante, España. $\triangle$ iD

${ }^{3}$ Graduado en Enfermería. Enfermero, Diócesis de Lodwar, Kenia. $\square$ iD

${ }^{4}$ Diplomado en Enfermería. Enfermero, Rotherham NHS Foundation Trust, Rotherham, Inglaterra. $\square$ (iD)

${ }^{5}$ Graduada en Enfermería. Magíster en Profesorado de Educación Secundaria Obligatoria y Bachillerato, Formación Profesional y Enseñanza de Idiomas. Enfermero, Clínica Vistahermosa, Alicante, España. \iD
RESUMEN El objetivo de este artículo es ofrecer una aproximación a la cultura de la tribu turkana del Triángulo de Ilemi, al noroeste de Kenia, en especial al modo en el que los miembros de este grupo étnico nómada entienden y dan forma a su propio constructo de salud y enfermedad. Se basa en un estudio etnográfico llevado a cabo en julio y agosto de 2014, en el que se realizaron observación participante en 15 aldeas de la tribu turkana, entrevistas informales y entrevistas en profundidad, con la colaboración de dos traductoras de la tribu y un enfermero. Se tomaron registros escritos, de video y de audio, los que se transcribieron para su posterior análisis. Entre los principales resultados de este estudio cabe destacar que tanto el concepto de salud como el de enfermedad distan mucho del enfoque occidental que a priori traen los proyectos externos y es probable que la falta de adherencia al seguimiento terapéutico de dichos proyectos esté relacionada con el desconocimiento cultural de ambos constructos por parte del equipo profesional.

PALABRAS CLAVES Antropología Cultural; Grupos Étnicos; Nómada; Proceso SaludEnfermedad; Kenia.

\begin{abstract}
This paper aims to provide a broad view of the Turkana tribe of the Ilemi Triangle in northwestern Kenya, especially regarding the way the members of this nomadic ethnic group understand and shape their own construct of health and disease. It is based in an ethnographic study carried out in July and August 2014, including participant observation of 15 villages of the Turkana tribe, formal interviews and indepth interviews carried out with the collaboration of two translators of the tribe and a nurse. Field notes as well videos and audio records were captured and transcribed for later analysis. Among the primary results of this study, it should be highlighted that the concepts of both health and disease differ greatly from the western approach that external projects bring with them a priori and it is likely that the lack of adherence to these projects is related to the scant cultural knowledge regarding such constructs on the part of the health professionals.
\end{abstract}

KEY WORDS Anthropology, Cultural; Ethnic Groups; Nomad; Health-Disease Process; Kenya. 


\section{INTRODUCCIÓN}

\section{Geografía y población del Triángulo de Ilemi}

Situado al este de África, entre Sudán del Sur, Etiopía y Kenia, se encuentra el Triángulo de Ilemi, una zona fronteriza de control keniano. Es considerada una de las zonas con mayor conflicto de África, debido a estar en continua disputa con los países colindantes por los frecuentes robos de ganado ${ }^{(1)}$. Cuenta con una extensión de entre 10.320 y 14.000 $\mathrm{km}^{2}$ y una geografía desértica, en la que la marginalidad económica, unida a la inestabilidad constante de los gobiernos de la región y la distancia que la separa de las zonas urbanizadas impiden que muchos de los beneficios de la medicina actual Ileguen hasta allí(2).

En Kenia, la población turkana está compuesta por seminómadas que viven en el valle superior del río Nilo y se extienden por el Gran Valle del Rift. Los turkana se distinguen por su estilo de vida y su cultura arraigada a sus tradiciones pastoriles. La posesión de ganado señala la riqueza que poseen, pero la finalidad de la cría no es la posterior venta sino el desarrollo de relaciones provechosas con otros turkana que también tengan ganado, los

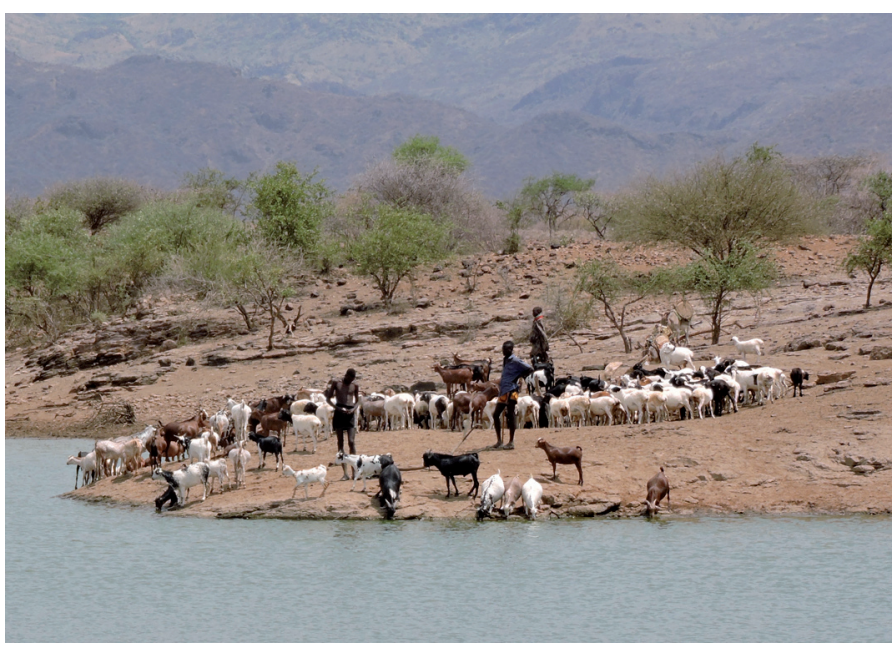

Figura 1. Pastoreo de cabras.

Tribu turkana, Triángulo de Ilemi, Kenia, 2014. eventos locales, danzas, sacrificios u ofrendas a su dios y/o intercambio por una o varias mujeres, puesto que la poligamia forma parte de sus costumbres ${ }^{(3,4)}$. El ganado es la principal moneda de intercambio y se utiliza, además, para alimentar a sus familias. Los pastores turkana dependen de las fuertes tormentas que se presentan con poca frecuencia en esta región, las cuales son la fuente principal de agua para el riego, la ganadería y el consumo humano (Figura 1). La extensión que compone esta región es de carácter desértico ${ }^{(4)}$ y las constantes sequías son parte del patrón climático. Diversos estudios ${ }^{(5,6,7)}$ muestran que las sequías son percibidas por los turkana como parte del hábitat en el que viven.

La movilidad es una estrategia de adaptación clave para mitigar el impacto de las sequías. Pese a esto, ante sequías severas, los pastores turkana pueden llegar a perder parte de su ganado, que es la fuente principal de sustento. Por esta razón, mediante el sistema de relaciones sociales, distribuyen parte de su ganado entre los que más lo necesitan a la espera de que, cuando pase la sequía, ambas partes salgan beneficiadas. A su vez, suelen verse obligados a desplazarse con el resto del ganado y su núcleo familiar hacia el sur, para poder aprovechar las precipitaciones que con mayor frecuencia aparecen en esa zona. Sin embargo, es posible que algunas tribus impidan su paso para robarles el ganado e incluso matarlos ${ }^{(4)}$. Como último recurso, y cuando la sequía es muy severa, los encargados del ganado pueden verse obligados a reducir el número de personas que se alimentan de este, puesto que junto a muchas familias turkana viven mujeres -cuyos esposos murieron o empobrecieron- y sus hijos son los que deberán reubicarse con otras familias mejor posicionadas o pedir limosna o trabajo en pueblos en los que existe actividad comercial.

En el territorio keniano existen alrededor de 28 clanes patrilineales distintos ${ }^{(8)}$, considerando un clan como el grupo de personas unidas por lazos de parentesco y los antepasados de un ancestro común. En cuanto al parentesco, incluye la adopción, el matrimonio y lazos genealógicos convencionales 
e incluso ficticios ${ }^{(9)}$. Estos grupos se distinguen de los habitantes que viven próximos a la ciudad de Nairobi (capital de Kenia), por ser grupos conservadores y arraigados a sus nobles tradiciones de pastoreo del ganado.

En las tierras que componen el Triángulo de Ilemi, la población de la tribu turkana está formada por tres de los 28 clanes mencionados. El antropólogo Marshall Sahlins ${ }^{(9)}$ define "tribu" como aquel grupo de personas que proceden de una familia o de la asociación de varias, que además comparten lugar de residencia en un territorio geográfico definido, con un antepasado común y suelen ser de la misma raza, creencias y costumbres. Los turkana se diferencian de sus tribus vecinas (niangatong, dassanesh, toposa, karamojong, samburu y pokot) porque no tienen líderes o representantes políticos formales, sino que sus líderes son los ancianos. La tradición indica que, si la situación lo requiere, los hombres ancianos se reúnen para la organización de redadas, presidir cualquier tipo de ceremonia o festejo, así como para tomar las decisiones importantes de la comunidad. Además consideran que, al igual que los ngimurok o profetas -término que se describirá más adelante-, los ancianos pueden influir en su dios Akuj, a través de ofrendas o sacrificios ${ }^{(10)}$.

Las mujeres son las encargadas de la crianza de los hijos, las obligaciones domésticas, el traslado de la familia y la construcción de una nueva cabaña o manyata si deciden emigrar hacia otro territorio. Manyata es como la gente de la tribu denomina a las cabañas hechas con barro, arcilla y ramas de acacia o de esekon, las dos variedades de árboles más abundantes de la zona (Figura 2).

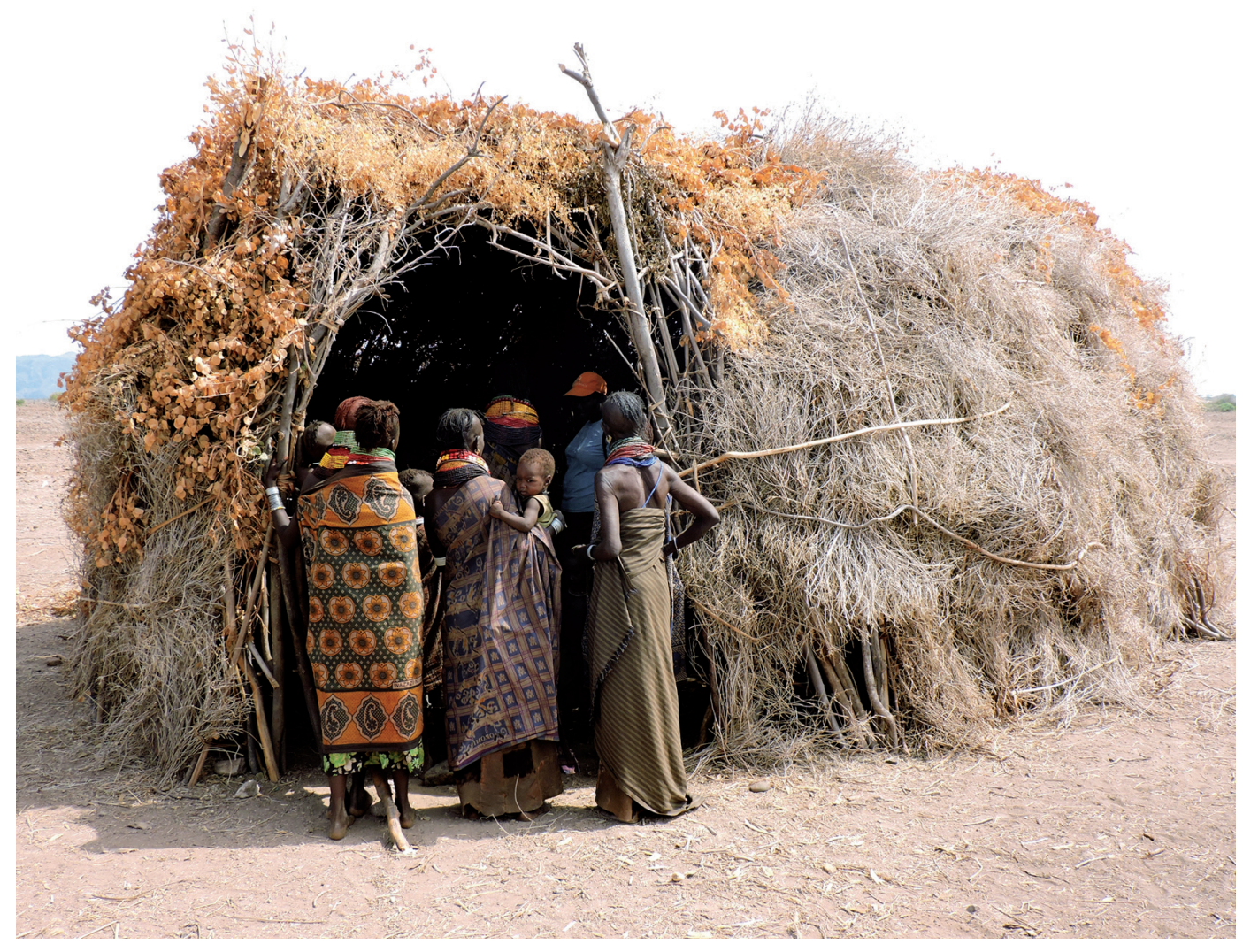

Figura 2. Manyata comunitaria (cabaña hecha con barro, arcilla y ramas de acacia o de esekon, creada para uso comunitario y reparto de alimentos de las organizaciones no gubernamentales). Tribu turkana, Triángulo de Ilemi, Kenia, 2014. 
La disparidad de género en la distribución de las tareas, que a menudo está profundamente arraigada en estas sociedades pastoriles, es un factor determinante en cuanto al acceso a los servicios de salud ${ }^{(2)}$. De hecho, no son las mujeres las encargadas de tomar decisiones respecto de cómo y cuándo ser atendidas por profesionales de la salud o del tratamiento requerido, sino que es el hombre el encargado de este tipo de decisiones.

Al igual que en la mayoría de las culturas ganaderas, los hombres son los encargados de los rebaños ${ }^{(11,12)}$, dirigen el ganado; se encargan de sacrificar a los animales y trocear su carne si se decide que forme parte de su dieta; defienden su rebaño y son grandes guerreros si tienen que defender sus bienes. Sin embargo, y a pesar de conservar las costumbres propias del grupo, la tribu turkana lleva años en contacto con visitantes del mundo occidental que llegan a ellos con proyectos relacionados con aspectos sociales y de salud, como se describirá a continuación.

\section{La vida en el Triángulo de Ilemi}

Los nómadas continúan la larga tradición iniciada por sus antepasados, que se basa en el traslado del ganado a terrenos con mejores condiciones para que este se alimente, crezca, se reproduzca y cubra sus necesidades de alimentación diarias. Cabras, camellos, burros y cebúes son los más utilizados como moneda de intercambio en diversas negociaciones, trueques y rituales. Aunque el hombre es el encargado del rebaño, cuando aún no está casado, la propiedad del ganado es asignada a la unidad familiar en la que vive, por lo que deberá compartir la carne o la leche con los hermanos. El matrimonio otorga la propiedad del ganado pero, para ello, y siguiendo la cultura turkana, hay que efectuar una especie de pago, que consiste en ofrecer una cantidad de ganado, previamente pactada, a la familia de la novia y futura esposa, lo que compensa de algún modo los años invertidos en su crianza y los que no podrá dedicar a la realización de labores para su familia al pasar a formar parte de la del marido. Como señalan algunas publicaciones referentes a la tribu turkana y sus tradiciones ${ }^{(10,13,14)}$, la poligamia es vista como símbolo de riqueza, puesto que quien más ganado posee mayores privilegios se puede permitir. Tal como defiende $\operatorname{Good}^{(15)}$ y retoma Gearhart:

...la universalidad de los marcos tradicionales de cuidados de la salud incorporan una gama de factores sociales, económicos, influencias espirituales y políticas de la salud de una persona ${ }^{(16)}$.

Es por ello que las unidades familiares realizan traslados continuos, impulsados por los periodos de sequía ${ }^{(17)}$ que fuerzan a que se desplacen para poder mejorar la posibilidad del pastoreo de sus rebaños ${ }^{(18,19)}$. Para conseguir el agua que se necesita a diario, tanto para el ganado como para sobrevivir, en algunas épocas tienen que andar durante más de una hora, y sustraerla de grandes pozos excavados manualmente en el lecho del río que, por la falta de lluvias, no ofrece agua en la superficie del cauce.

El matrimonio juega un papel fundamental en la organización social turkana, creando alianzas y redes de apoyo entre los clanes, dado que las mujeres pasan a formar parte del clan del marido y los hombres no pueden casarse con una mujer de su propio clan, es decir, del clan de su padre. Los matrimonios son pactados entre las familias, una de las cuales paga una dote proporcional a la riqueza que posea: por ser una familia pudiente o por haber robado el ganado a tribus vecinas. Las grandes sequías pueden ser un factor clave para que el responsable de la familia tome una decisión tan importante como es el matrimonio de una de sus hijas, tanto con el objetivo de compensar las posibles pérdidas de ganado en la época de sequía, como para aumentar los lazos sociales con otros turkana. Los matrimonios pactados suelen presentar casamientos de niñas hasta de 12 años con varones de 60 años, por lo que las dotes entre los turkana son de las más altas que se conocen entre las poblaciones pastoriles ${ }^{(4,20)}$. 
A pesar de que los turkana conocen la medicina occidental que el hombre blanco introdujo en el pasado, tal como señala Bayardo et al. al citar a Soustelle, las nociones y las prácticas relativas a la enfermedad y a la medicina son "una mezcla inextricable de religión, de magia y de ciencia"(21). Los turkana creen en un dios a quien conocen como Akuj, y al cual atribuyen el fenómeno de la lluvia: si Akuj es feliz lloverá y si, por el contrario, está enfadado con los habitantes, no lloverá. Entre los diversos personajes en los que el pueblo turkana confía para que su dios no se enfade y los prive de la lluvia están los ngimurok, personas que transmiten lo que su dios les comunica en sueños. Equivalen a lo que otros grupos denominan como adivinos o profetas, los cuales son capaces de predecir o interpretar lo que su dios les transmite, para posteriormente comunicarlo a su pueblo. Los ngimurok emplean una gran gama de conocimientos sobre la naturaleza que los rodea, como la humedad ambiental, la temperatura, el desplazamiento de las aves o insectos, etc. Para mejorar la subsistencia de los turkana se necesitan buenas condiciones climáticas para que, en sus tierras áridas, broten alimentos para su ganado.

Según sus creencias, consideran a su dios como algo sagrado, que puede ayudarles en tiempos de necesidad o en la curación. A cambio de obtener este tipo de ayuda, los turkana suelen sacrificar parte de su ganado a modo de ofrenda. Como señala uno de los informantes privilegiados basado en su experiencia y conocimientos, también utilizan este método porque creen que los ayudará en la recuperación de las enfermedades que los occidentales conocen habitualmente como de larga evolución (brucellosis, leishmaniasis, enfermedades cardíacas, etc.) porque en muchas ocasiones las relacionan con una especie de maleficio que describen como algo similar al "mal de ojo" de otras culturas, es decir, la creencia "que atribuye a la mirada de algunas personas y a determinados actos o sentimientos negativos la capacidad de causar daño a otros" ${ }^{\prime 22)}$.

\section{Proyecto clínica móvil}

En Kenia, a comienzos de la década de 1990, la African Medical and Research Foundation (AMREF) tenía más de treinta años de experiencia con unidades móviles de atención a la salud entre pastores kenianos ${ }^{(23,24)}$. Las clínicas móviles fueron creadas para combatir la dispersión geográfica entre las poblaciones pastoriles y los servicios de salud, lo cual no significa que se haya creado un lazo con los proveedores de servicios, debido a la diversidad de preferencias de los nómadas por las medicinas tradicionales que podían encontrar en los diversos sanadores tradicionales de sus tribus ${ }^{(2)}$.

A pesar de haber existido con anterioridad un proyecto de atención médica a cargo de la AMREF en el noroeste de Turkana para los masáis ${ }^{(25)}$, se crea el proyecto clínica móvil de la mano de dos enfermeros españoles que residen en la misión de Lobur ${ }^{(26)}$, la cual se encuentra situada en la zona de Lobur y cubre un territorio de unos $15.000 \mathrm{~km}^{2}$ en el Triángulo de Ilemi. La misión de Lobur es una asociación pública de fieles cristianos de la Iglesia Católica, integrada por sacerdotes y laicos, hombres y mujeres, de diversas partes del mundo, y es la coordinadora de todos los proyectos de desarrollo que la Missionary Community of St Paul the Apostle (MCSPA) lleva a cabo en la región de Turkana. El proyecto clínica móvil abarca 12 aldeas, de las cuales cuatro corresponden a centros de atención materno-infantil establecidos por la MCSPA y otras ocho a poblaciones nómadas. El proyecto planea extender sus servicios a unos 22.750 beneficiarios directos y a otros 12.000 indirectos $^{(13)}$.

La clínica móvil está compuesta por dos enfermeras, dos traductoras autóctonas y un clinical officer, que es uno de los principales proveedores de servicios en este país y tiene licencia para realizar atención de rutina en medicina general, especialmente a nivel de atención primaria ${ }^{(27)}$. Para el desplazamiento por el desierto se utiliza un vehículo todo terreno, el que diariamente se equipa con dos grandes baúles repletos con la medicación que se necesitará en la zona a visitar, y con las 
pocas herramientas diagnósticas de las que se dispone: fonendoscopio, esfingomanómetro, pulsioxímetro, test de malaria y VIH, set diagnóstico de otoscopio y oftalmoscopio.

El equipo sanitario se desplaza con la clínica móvil para tratar de brindar atención a las poblaciones más alejadas, con la intención de llegar a los hogares de los propios enfermos y, si la situación lo requiere, trasladarlos al hospital de la capital en Lodwar (a unas siete u ocho horas de viaje en vehículo de doble tracción, si las condiciones meteorológicas son favorables). En el proyecto se incluye la prevención y concienciación sobre la promoción de la salud y los hábitos saludables e higiénicos, preparación al parto grupal por parte de matronas, controles a las gestantes, así como formación de personal sanitario local, con el fin de asegurar la continuidad y sostenibilidad del proyecto por personal sanitario autóctono.

Napeikar es uno de los poblados con mayor población agrupada (unas 2.500 personas) a la que se atiende 2 o 3 días a la semana. Dicha atención es posible debido a la proximidad con la misión de Lobur, centro logístico y residencia de los integrantes sanitarios de la clínica móvil, así como el lugar donde se iniciaron diversos proyectos, como un colegio infantil (los niños reciben educación hasta los 10 o 12 años puesto que sus padres posteriormente les asignan diversas responsabilidades familiares), talleres para embarazadas o la clínica móvil. La continua atención prestada permite un rápido acceso al trabajo de campo, a la observación participante, y posibilita entrevistar a la gran mayoría de sus integrantes.

Una de las traductoras que compone el equipo del proyecto clínica móvil vive en dicha aldea, es autóctona, tradujo las entrevistas de este estudio y también fue entrevistada. Tener a esta persona de enlace con los turkana ha sido de gran ayuda puesto que, por un lado, es de su propia tribu y de género femenino, lo que facilita la accesibilidad a la mayoría de las candidatas mujeres entrevistadas, tanto por ser de su propio género como por la mayor demanda de atención de estas, dado que es la mujer la que ejerce el rol de cuidadora en la familia, en materia de salud y enfermedad. Por otro lado, es conocedora de las características que poseen los sujetos que van a ser entrevistados, el lugar y el momento más adecuado para que sus declaraciones no estén condicionadas por cualquier tipo de influencia.

\section{METODOLOGÍA}

El propósito del estudio fue intentar conocer el concepto salud y enfermedad de los nómadas turkana para, posteriormente, poder planificar una interacción terapéutica culturalmente congruente y competente, que permita mejorar la eficacia de las intervenciones externas y ayude a la mejora en la aplicación de los cuidados en salud y enfermedad de la tribu.

\section{Diseño de la investigación}

Dentro de la metodología cualitativa, se ha elegido el enfoque etnográfico que permite realizar un estudio directo de personas y grupos durante un cierto periodo de tiempo $^{(28)}$ y en un momento específico del desarrollo de este grupo cultural, desde la perspectiva de los propios actores, incluyendo su cosmovisión. Concretamente, se utilizó la observación participante a través del diario de campo y las entrevistas etnográficas para conocer el comportamiento social, registrar una imagen realista y fiel del grupo en estudio y comprender la forma en la que el grupo usa sus entornos naturales, se organiza, estructura socialmente las diferentes facetas de su día a día y su relación con otros grupos o sectores de la sociedad ${ }^{(3,4,17,18,19,29,30)}$. Específicamente, se buscó conocer cómo la tribu turkana interpeta el concepto salud y enfermedad con la finalidad de tener una visión más acertada y así poder intervenir con mayor eficacia en materia de salud y enfermedad, aplicando a posteriori cuidados culturalmente competentes y congruentes con dicha concepción. 


\section{Determinación de las técnicas}

Para la recopilación de datos se utilizó tanto la observación participante como la observación directa (en los primeros días de acceso al campo por parte del investigador principal), así como la entrevista informal o conversacional e individualizada a miembros de la tribu, en la que colaboraron diariamente dos traductoras de la tribu y un enfermero que formaba parte del proyecto clínica móvil, todos ellos informantes privilegiados de este estudio. También se realizaron entrevistas en profundidad a distintas personas consideradas informantes secundarios, de habla hispana e inglesa, las cuales vivían y compartían experiencias con los nómadas desde hace al menos cinco años. La observación participante se hizo en 15 de las aldeas que componen la tribu turkana, las notas se plasmaron en un diario de campo a través de registros escritos, de video y de audio. En todas esas aldeas se realizaron entrevistas informales a 42 nómadas, seleccionados por las traductoras autóctonas y el clinical officer a partir de la predisposición a participar. De los entrevistados, un 78,6\% eran mujeres frente a tan solo el $21,4 \%$ de hombres, ambos de edades comprendidas entre los 18 y los 50 años según refirieron los propios participantes. Entre los 42 nómadas, con la ayuda de la traductora, se seleccionaron a aquellos que podían aportar mayor disponibilidad e información, y luego de explicitar que su relato sería utilizado para un estudio de investigación y que su identidad sería resguardada, se llevaron a cabo 14 entrevistas en profundidad de unos 90 minutos aproximadamente.

Durante las entrevistas se constató que las mujeres eran mucho más accesibles que los hombres y aportaban más datos, hecho que los informantes privilegiados habían mencionado de antemano, ya que se consideran las representantes y cuidadoras de la familia, por ello frecuentan más el poblado que los hombres, quienes suelen ejercer otras labores más alejadas de donde la clínica móvil ofrece atención. A su vez, los integrantes de la misión de Lobur (como los dos sacerdotes que la dirigen y las seis personas que más experiencias han tenido en dicho territorio) también fueron entrevistados, complementando la información recopilada, puesto que han interactuado durante muchos años con la tribu y conocen las tradiciones, cultura y creencias que acostumbran a seguir, convirtiéndose de este modo en informantes secundarios del estudio. En la observación, tanto participante como directa, se ha podido acceder a más del $70 \%$ de la población que abarca el proyecto puesto que, a pesar de existir grandes distancias, el programa diario permite la interacción con todos los poblados que no hayan cambiado de ubicación al menos una o dos veces al mes. Se contó, como informante privilegiado principal, con un enfermero español quien permaneció los tres meses de verano entre 2008 y 2012 y, posteriormente, pasó a ser componente del equipo sanitario desde julio de 2012 hasta diciembre de 2014, de modo que disponía de la experiencia directa tanto por el trato diario con las madres y familias turkana, como por el seguimiento de casos con diversos problemas.

Paralelamente, se ha recopilado información a través de una revisión bibliográfica en distintas bases de datos y catálogos bibliográficos, así como tesis doctorales e información procedente de bibliografía gris que fue contrastada en diferentes blogs.

Cabe hacer una importante mención a la creación de lazos con los nómadas, en forma previa a la entrada a campo, hecho que facilitó la introducción de los investigadores en su contexto diario, así como la recopilación de datos, sin que nuestra presencia, especialmente la del investigador principal, pueda suponer un efecto negativo al intervenir en sus vidas.

\section{Acceso al ámbito de la investigación}

Como integrante del equipo sanitario del proyecto clínica móvil, el investigador principal pudo interactuar en forma directa con la población turkana, tanto en la atención como en las entrevistas realizadas en julio de 2014. 
La programación pactada con los organizadores del proyecto con respecto a la selección del escenario para atender a las distintas aldeas turkana sufría modificaciones justificadas ante imprevistos que podían incluir desde un problema con el vehículo, que los integrantes de una aldea emigraran a otra zona sin aviso alguno, o que el caudal de un río aumentara en época de lluvia impidiendo el acceso, entre otros. La integración al escenario fue facilitada informalmente gracias a que uno de los investigadores formaba parte de la clínica móvil e iba acompañado de un enfermero, un clinical officer y una traductora que realizaban esta labor desde agosto del año 2012.

\section{Selección de los entrevistados}

La selección de los informantes primarios se basó, en su mayoría, en mujeres turkana, con edades entre los 18 y los 50 años, puesto que son las que se encargan del trabajo físico, los hijos, las labores de la manyata, la comida, etc. Los hombres suelen encargarse del rebaño y la organización de la distribución geográfica de la familia, según la fertilidad que las tierras ofrezcan a su ganado, por ello son menos accesibles y frecuentan mucho menos el escenario elegido. Para las entrevistas, tanto las traductoras como el enfermero seleccionaron a aquellas personas más colaboradoras y predispuestas habitualmente a hablar con ellos, evitando de este modo posibles conflictos culturales que pudiesen perjudicar a corto y mediano plazo el desarrollo de las tareas sanitarias de la clínica móvil, aun a sabiendas de que esta decisión podría incurrir en un potencial sesgo en el tipo y calidad de la información.

\section{Recogida y procesamiento de los datos}

Cada día se registraban notas de campo in situ. Tras llegar a la base de Lobur, lugar donde residían algunos de los integrantes de la clínica móvil, se codificaban y registraban dichas notas y, a la vez, se contrastaban con los datos de días anteriores para reflexionar y mejorar el análisis de forma continua, así como para crear nuevas hipótesis basadas en los fenómenos culturales o patrones conductuales observados.

\section{Aspectos éticos de la investigación}

Todos los participantes fueron informados previamente de los objetivos de la investigación, así como de su carácter voluntario y anónimo. Debido al alto nivel de analfabetismo de la zona, los entrevistados aceptaron el consentimiento informado verbalmente, luego de que la traductora les leyera el documento con los términos de participación en el estudio, hecho que quedó grabado. Durante este proceso, se contó con la presencia de al menos dos entrevistadores, o un entrevistador y un facilitador, quienes además de actuar como testigos les explicaron en su propio idioma que la aportación de sus relatos sería utilizada para el desarrollo de una investigación, en la que se respetarían la privacidad y el anonimato.

\section{RESULTADOS}

\section{Creencias en salud y enfermedad}

En Turkana, sus habitantes recurren a distintos tipos de sanadores según la causa que ellos le atribuyan al problema que ha aparecido. Mantienen una fuerte y arraigada creencia respecto al conjunto de habilidades únicas que poseen el hechicero, la bruja, el emuron y el ekerujan, ya que predicen, adivinan y tratan los sucesos que se dan en esas tierras.

Entre los encargados de sembrar el mal entre los habitantes y sus rebaños se encuentran los ngikasubak, los cuales utilizan en secreto diversos conjuros en contra de la sociedad, y los ngikapilak que son aquellos que se especializan en la invocación de fuertes maldiciones utilizando partes del cuerpo de los recién fallecidos. Podríamos establecer un paralelismo con lo que otros 
autores denominan bruja y su conocido mal de ojo ${ }^{(22)}$, como es el caso de Shankar y Haverkort ${ }^{(3)}$ quienes definen el mal de ojo como "la mirada nociva de una persona hacia un animal joven", que podría "contraer enfermedad en venganza por conflictos humanos", como celos o envidias, y que puede ocasionar escozor, hinchazón, heridas y edemas. Debido a sus creencias y tradiciones el hechicero juega un papel fundamental en las aldeas, suele ser la persona de referencia a la que acuden los componentes de la tribu como principal sanador de determinadas patologías (habitualmente crónicas), el cual emplea sus conocimientos sobre algunas plantas medicinales acompañadas de conjuros, ritos y ceremonias para espantar los males y los demonios.

El emuron (singular) y los ngimurok (plural) $^{(30}$ p.168), hacen referencia a los curanderos locales, que expresan lo que su dios les transmite en los sueños. El misionero antropólogo Barrett ${ }^{(31)}$ sostiene que los "adivinos verdaderos", también conocidos como los "adivinos de dios", son los más respetados de los ngimurok porque reciben revelaciones directamente de Akuj, normalmente a través de los sueños. También sostiene que utilizan otros medios como la lectura de los intestinos de un animal sacrificado, el tabaco, cuerdas, calabazas, piedras, y el más famoso de ellos es el lanzamiento de sandalias: la forma en la que caen al suelo puede ser interpretada como una señal ${ }^{(31)}$. Entre las funciones por las que los turkana solicitan su ayuda está la de descubrir la causa, tratamiento y cura de enfermedades, las sequías, inundaciones, epidemias $\mathrm{u}$ otros desastres que no puedan controlar. A cambio, los ngimurok solicitan diversos sacrificios de ganado con la finalidad de aplacar o influir en los actos de Akuj, para que no esté enfadado con el pueblo y les depare condiciones adversas y/o desastrosas.

El ekerujan o soñador (el profeta) es la forma más poderosa de emuron, sus sueños tienen mayor claridad y Akuj les habla en ellos. Se dice que este tipo de emuron es el más poderoso y que en su iniciación fue arrebatado y llevado ante Akuj, por lo que a partir de ese momento pasa de ser humano a adquirir la facultad del emuron más poderoso: el ekerujan.

Los ngimurok suelen ser hermanos, puesto que esa cualidad se transmite de padres a hijos. Cabe aclarar que los ngimurok son simples transmisores de lo que Akuj les habla en los sueños, por más que realicen rituales o sacrificios, nada se pueden hacer si su dios decide que haya sequías, plagas, enfermedades, etc. Estos simplemente transmiten lo que interpretan que Akuj les dice mientras duermen, y solicitan sacrificios para disminuir la ira de su dios y no ser castigados por este.

\section{Salud como interacción social}

Entre los resultados obtenidos en las diversas entrevistas realizadas a la población en estudio, encontramos un gran consenso respecto de la percepción sobre la salud, la cual estaría directamente relacionada con la familia, la tribu, el entorno y la fe en su dios, es decir que es fruto de la interacción con todo lo que los rodea y en ningún momento es percibida como un bien particular e independiente de dicha interacción social. En las distintas entrevistas, se hacía referencia a ella como la facultad de seguir adelante en sus tareas diarias, que no se les prive de poder cumplir sus obligaciones pastorales, corresponder a su familia, así como tener a su dios contento para que no les depare lo peor. Esta concepción no menciona el bienestar físico individual, por lo cual tener dolor, una enfermedad crónica u otra problemática que no les impide en gran medida cumplir con lo anteriormente mencionado, no lo considerarán como falta de salud, puesto que no los priva de la ejecución de lo más importante para ellos que es el pastoreo de su ganado, la familia y sus creencias.

\section{Caso de ejemplo}

Encontramos un grupo de jóvenes que jugaba junto a un arma de mediano calibre cuando, a causa de un tropiezo, esta se disparó alcanzando a uno de ellos. Por lo general, 
no es extraño que la mayoría de los pastores porte un arma de fuego como la AK-47 o una Kalashnikov ${ }^{(1)}$, tanto para la defensa de sus ganados como para los posibles robos o agresiones que estos conllevan (Figura 3), pero en esta ocasión el desafortunado incidente alcanzó a un joven. Como en todas las sociedades, los incidentes anómalos se extienden rápidamente entre los habitantes de la zona, por lo que el equipo sanitario tardó cinco días en enterarse del amargo incidente, así como de la dulce noticia de que el joven estaba con vida. Tras buscar a la traductora y preguntar a diversos nómadas si conocían el paradero del afectado, lo encontramos caminando con un bastón, cojeando y con un sucio vendaje casero hecho de tela en la pierna afectada. Al acercarnos, el joven se encontraba sentado bajo la sombra de una manyata, tenía un aspecto semejante a cualquiera de los que habitaban el poblado, pero cuando intentó movilizarse los gestos de su cara delataron el dolor que sufría. A través de la traductora le preguntamos qué le había pasado, cómo se encontraba, por qué estaba allí, etc. A lo que respondió:

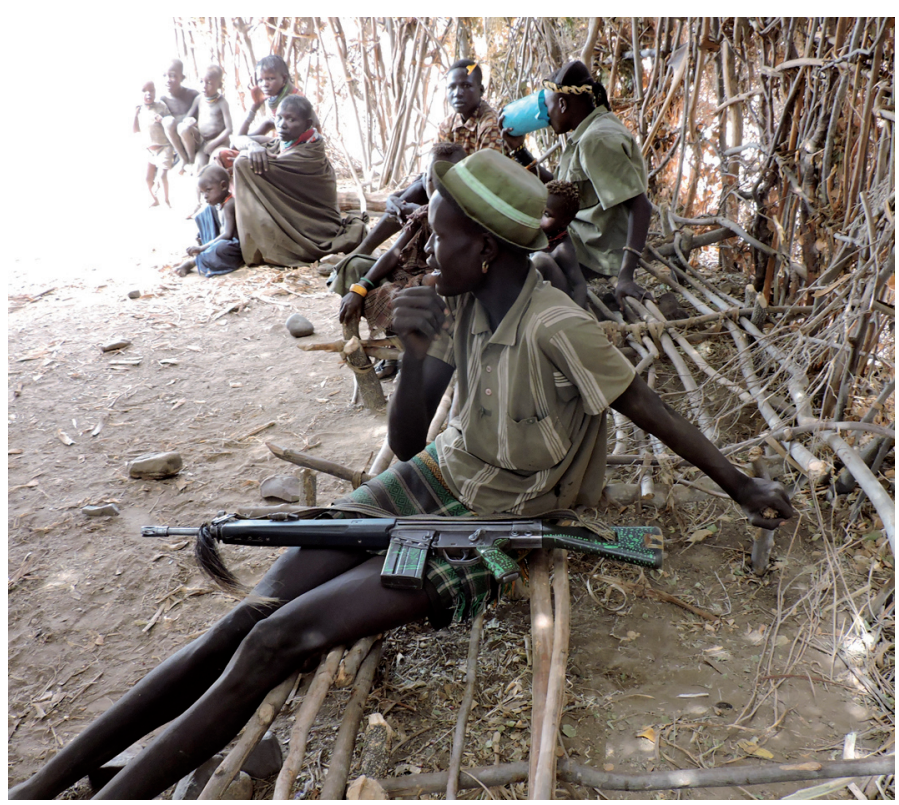

Figura 3. Joven encargado del pastoreo de cabras. Tribu turkana, Triángulo de Ilemi, Kenia, 2014.
...Hace cinco días me pasó esto, yo jugaba con mis amigos y por lo visto alguna bruja me habrá echado un mal de ojo, fui al hechicero y me puso esto, me sigue doliendo pero puedo andar con ayuda del bastón y pronto tengo que reemprender mi trabajo de pastoreo con el rebaño que mi padre me ha asignado...

Tras destapar el sucio vendaje, observamos que se trataba de una herida con apertura mínima de entrada por la parte frontal del hueso tibial, y salida por la parte lateral externa a ese mismo nivel. Afortunadamente, no había indicios de que hubiese tocado el hueso, pero el orificio de salida presentaba un desgarro de unos 5 o $6 \mathrm{~cm}$ de diámetro, con signos de infección, moscas posándose en la herida y sin sangrado. Rápidamente se procedió a realizar la cura de la herida y la administración de antibiótico. En los siguientes cuatro días se acudió al lugar para administrar la medicación y curar la herida. Llegado el quinto día, el afectado había emigrado sin dar explicaciones previas al equipo que lo atendía. Según pudimos saber, la familia había decidido recoger sus cosas y trasladarse a tierras más fértiles para sus ganados, lo cual se podría interpretar desde diferentes puntos de vista sobre la base de lo manifestado por los informantes: a) su hijo ya podía andar mejor y no necesitaba mayor ayuda; b) el mal de ojo ya había pasado; c) la priorización del interés familiar a la del beneficio de uno solo de sus integrantes; d) la selección natural determinaría que el más fuerte sobrevive y el débil no.

\section{Enfermedad como maldición o hechizo}

A diferencia del concepto salud, la enfermedad es interpretada como aquello que no les permite o les dificulta acceder a sus obligaciones cotidianas. Algunos relacionan estrechamente la causa de la enfermedad con el olvido de los antepasados, la ofensa a su dios o la brujería, por lo que recurren a oraciones y sacrificios para curar la relación con 
los antepasados. Sin embargo, cuando observan algún problema o alteración en la salud de la población infantil no lo relacionan con una maldición puesto que un niño no puede haber ofendido a su dios aunque sí puede haber sido víctima de brujería. Así lo describía el informante privilegiado cuando mencionaba el caso de algún niño que presentaba alguna anomalía física o psíquica como, por ejemplo, la epilepsia. La mayoría de las familias consideran que la epilepsia no es una maldición y, para buscar una solución, suelen pedir ayuda al personal sanitario tras haber llevado a cabo las oraciones y sacrificios que pudieran eliminarla. También existen casos poco habituales de niños diagnosticados de autismo, obviamente con el comportamiento típico de la patología, que es percibido como anormal o distinto por el resto de la sociedad. Según constataron los informantes, estos casos suelen ser interpretados por parte de la tribu como consecuencia de actos anormales en la relación de pareja de los padres, como es el caso del canibalismo o la necrofilia, lo que lleva a la tribu a pensar que son niños malditos como parte del castigo o maldición por el comportamiento de los padres.

Como se ha mencionado anteriormente, el cuidado de los niños recae en la familia y cuidan de ellos sea cual sea su problema, con la esperanza de que algún día se solucione, ya sea por sí mismo o por factores externos. Pero hay que tener en cuenta el factor supervivencia en las tierras desérticas en las que residen, por lo que, como anteriormente se ha mencionado, cuando las condiciones son desfavorables y los recursos escasean, aparecen periodos de hambruna y el instinto de supervivencia afecta a los más débiles, es decir, aquellos que viven acogidos en el núcleo familiar, como mujeres cuyo esposo murió o empobreció y sus hijos. Así pues, si en una familia nacen gemelos en una mala época, dejarán de alimentar al que consideren más débil. A los ancianos incapaces de mantenerse por sí mismos les pasará lo mismo, al igual que las mujeres y los hijos serán apartados de la comida de menor a mayor. Los más débiles siempre son los primeros en ser apartados de la comida, por considerar que tienen menos posibilidades de supervivencia.

\section{Caso de ejemplo}

Encontramos a una mujer turkana de unos 50 años, alejada de Lobur pero dentro del campo de acción de atención sanitaria. Esta mujer tenía cáncer de cuello de útero terminal. Se había mudado a uno de los poblados más alejados de cualquiera de los posibles dispensarios (lugar donde se atiende a los habitantes enfermos de la zona, y reciben atención sanitaria y farmacéutica sin estar hospitalizados, así como primeros auxilios a pacientes heridos). Con este gesto dificultaba la posibilidad de paliar el dolor producto de la fase en la que se encontraba su enfermedad, además del cuidado que implicaba la sonda suprapúbica que portaba. La actuación del equipo sanitario buscaba razonar junto al paciente para que se trasladara a un poblado más cercano como es Napeikar, para así poder realizar su atención diaria, puesto que hay una agenda que seguir para poder atender toda la demanda. Frente a ello, la señora argumentó lo siguiente:

Señora: -No quiero irme de aquí, no hay nadie de mi familia y las brujas las oigo por las noches cerca del poblado.

Enfermero: -¿Qué te pueden hacer las brujas?, zacaso ellas tienen poder de hacerte algo?, ¿sabes que nos resulta muy difícil venir aquí y no podemos cuidarte tan bien como en Napeikar?

Señora: -Sí, sobre todo una vez muera, y voy a morir pronto, desenterrarán el cadáver y se lo comerán, y no quiero que eso me pase, por eso no puedo volver a Napeikar, porque las brujas siguen allí.

Enfermero: -Pero, ¿crees en dios?, ¿tú crees que dios te dejará sola ante las brujas?

Señora: -Sí, claro que creo en dios, en tu país tal vez las brujas no te puedan hacer nada, pero aquí sí y no quiero que se coman mi cadáver, por eso no voy a volver a Napeikar, porque las brujas están allí. 
Enfermero: -Vamos a plantear una solución, aquí no podemos tratar el dolor ni la limpieza de tu herida, no podremos controlar que te duela lo menos posible, ¿qué te parece si te trasladas a Meyen?, también está cerca y así podemos venir a verte más a menudo.

Señora: -¿Meyen?, solo con la condición de que me asegures que no hay brujas que se puedan comer mi cadáver cuando muera.

Enfermero: -Te aseguro que no las hay, vale pues en un día te vendremos a buscar para llevarte a Meyen, ¿te parece bien? Señora: -¿Por qué te preocupas tanto si sabes que voy a morir?, pero vale, iré a Meyen.

El miedo a las brujas y a lo que pudiesen hacer con ella después de muerta asustaba tanto a la paciente que no era capaz de valorar que sin los cuidados y el control del dolor era posible que su tiempo de vida se redujera, acelerando así el proceso para que las brujas se comieran su cadáver. También confirma la teoría del significado que le dan al concepto de enfermedad, puesto que ella se consideraba enferma porque el dolor y la sonda que porta no le permiten realizar su rutina diaria, además interpreta que la brujería ha tenido relación con el evento, e incluso la seguirán acechando después de muerta.

\section{CONCLUSIONES}

La tribu turkana lleva muchos años compartiendo su forma de vivir con diversos visitantes que llegan con diferentes intenciones y/o propósitos, como las organizaciones no gubernamentales (ONG) que tratan de combatir la desnutrición, los proyectos como el de las clínicas móviles o los que, desde antaño, lleva a cabo la iglesia católica para mejorar los recursos de estas poblaciones ${ }^{(23,24,25)}$. Este continuo contacto ha sido, posiblemente, el impulsor de la confianza creada hacia las personas de piel blanca dada la intención de mejorar sus recursos puesto que, a pesar de que el gobierno del país es el encargado del suministro de parte de dichos recursos y medicamentos, normalmente son extranjeros los que desarrollan diferentes proyectos para la atención de las poblaciones más alejadas de la civilización. Por lo tanto, el "blanco" forma parte de su hábitat y hasta el momento ha demostrado tener habitualmente "buenas intenciones", según se pudo constatar durante las entrevistas.

Tener acceso a la medicina occidental no siempre es un beneficio que esté al alcance de todos los turkana puesto que, además de la distancia, su sistema social y económico en muchas ocasiones no les facilita el acceso. Hace años que las clínicas móviles (tanto de proyectos occidentales como del propio país) permiten llegar a diversos territorios de difícil acceso para los recursos sanitarios, por lo que facilita que los nómadas elijan su uso y no tengan que seguir dependiendo exclusivamente de las prácticas en salud tradicionales de la cultura ${ }^{(25)}$. Al igual que Sandford ${ }^{(32)}$ y Swift ${ }^{(33)}$ en sus estudios realizados sobre la organización de los servicios sociales para los pastores y la prestación de servicios para personas nómadas, observamos que los movimientos impredecibles de los nómadas, la mala comunicación y la baja densidad de algunas poblaciones dificultan la atención y aumentan los costos en comparación a los servicios estáticos que más fácilmente pueden atender a los no nómadas. Además, no se les puede realizar un adecuado seguimiento y en muchas ocasiones no entienden la importancia de finalizar un tratamiento, es decir, no entienden que no consta solo de una parte y que tengan que volver para completarlo.

Por otro lado, cuando deciden ser atendidos y su diagnóstico necesita tratamiento, no todos los fármacos son entregados a su demandante. Las embarazadas, niños y ancianos reciben los medicamentos que el sanitario valora que necesitan sin ningún intercambio de bienes; sin embargo, tanto a mujeres como a hombres jóvenes se les impone una especie de pago simbólico por la medicación, el cual puede consistir en la entrega de una pieza de ganado, artesanía (cuencos, vasijas, taburetes 
Ilamados ekicholong, etc.), o abalorios que utilizan tanto para mostrar la riqueza de su familia como para la decoración de sus cuerpos $^{(15)}$. Es interesante destacar la importancia que juega este tipo de trueque, así como la negociación del intercambio, puesto que la finalidad no es en sí el pago por el fármaco sino el hecho de que no se haga un uso abusivo de los recursos o una posible reventa posterior. Estos trueques posibilitan la regulación de los escasos recursos de los que dispone el territorio del Triángulo de Ilemi, y disminuyen el consumismo de parte de los integrantes de esta tribu, que con el paso del tiempo han comenzado a llevar una vida un poco más dependiente de las facilidades que aportan los proyectos destinados a ellos.

Harragin ${ }^{(25)}$ refiere que, en el estudio que realizó durante tres años sobre la disposición de asistencia sanitaria en el noreste de Kenia, no todos los nómadas que acudían a la clínica móvil lo hacían por estar enfermos: algunos decían sentir los síntomas de algún integrante de la familia que sí tenía la sintomatología, otros referían enfermedades del pasado para anticipar una posible recaída, o incluso para almacenaje, reventa y/o tratar a sus animales.

El cumplimiento del tratamiento pautado está íntimamente relacionado con las creencias que los turkana guardan hacia Akuj y los distintos representantes de este, así como con la educación recibida y las experiencias previas (positivas o no) con el personal sanitario. Así, cuando se trata de enfermedades crónicas con mal pronóstico pueden suceder distintas situaciones:

1. Que acudan directamente a buscar atención sanitaria y que ante la falta de recursos o la avanzada evolución de la enfermedad no se les pueda ofrecer una solución, por lo que a posteriori recurrirán al hechicero.

2.Que al tratarse de algo cuya evolución arrastran hace mucho tiempo lo relacionan con un mal de ojo realizado por las brujas por lo que, en primer lugar, buscarán la atención del hechicero y, posteriormente, la del personal sanitario si el hechicero no los pudo ayudar. Otros deciden acudir al personal sanitario en primera instancia, pero debido a la falta de rapidez en la obtención de resultados a través del tratamiento pueden abandonarlo o solicitar la ayuda de los sanadores turkana.

3. También se encuentran casos de nómadas que, debido a la lejanía de sus poblaciones, recurren directamente al hechicero por no poder optar por el personal sanitario.

4. Hay otros nómadas que deciden seguir tanto el tratamiento sanitario como el del hechicero, lo cual puede ser altamente perjudicial debido a la posible interacción o sobredosificación de alguno de los componentes de los tratamientos. Cuando todos los recursos disponibles fracasan, los turkana suelen acudir al emuron con la esperanza de que los pueda ayudar con sus poderes sobrenaturales. Cabe destacar que, debido a las distintas formas de interacción de cada individuo con la enfermedad, habrá que valorar múltiples aspectos para encauzar la mejor adherencia al tratamiento, debido a que son diversas creencias las que condicionan que este se siga o no. Este entramado o red de toma de decisiones en materia de salud y enfermedad se ha ido tejiendo a lo largo de los años sobre la base de la experiencia vivida por la tribu y la incorporación de cada vez más opciones terapéuticas, lo que ha llevado a configurar un abanico de servicios cada vez más complejo, en el que se entremezclan características culturales propias o tradiciones del grupo con otras, externas, traídas del mundo occidental, que han dotado a la tribu de cierta capacidad de elección que anteriormente no existía. El éxito en el resultado curativo obtenido y el tiempo de resolución para llegar a la curación han ido marcando las decisiones que toman los miembros de la tribu. Sin embargo, parece que la percepción de los constructos tradicionales de salud y enfermedad de la tribu se ha conservado, como se puede apreciar en estudios etnográficos en sociedades urbanas cuyas conclusiones no distan demasiado de las expresadas aquí. Así, en el estudio de Lillo $^{(34)}$ se muestra que aunque una población pueda incorporar opciones, servicios o intervenciones distintas a las 
tradicionalmente establecidas, prevalece la percepción de la salud y la enfermedad culturalmente aprendida. En realidad, es el grupo profesional el que debe conocer la cultura y los constructos en materia de salud y enfermedad de la población a la que va a tratar, para poder llevar a cabo un abordaje culturalmente congruente ${ }^{(35)}$ y con un enfoque de mejora sobre la situación del grupo ${ }^{(36)}$.

Cabe destacar el hallazgo sobre la definición de salud para la tribu turkana que aporta este estudio como: la facultad de seguir adelante en sus tareas diarias, poder cumplir sus obligaciones con respecto a la tribu, corresponder a su familia, así como tener a su dios contento para que no les depare lo peor. A la vez, la enfermedad es interpretada como: aquello que no les permite o les dificulta acceder a sus obligaciones cotidianas, algunos relacionan estrechamente la causa de la enfermedad con el olvido de los antepasados, la ofensa a su dios o con la brujería, por lo que recurren a oraciones y sacrificios para subsanar dichas causas. Ambos conceptos distan mucho del enfoque de salud y enfermedad occidental que a priori traen los proyectos externos como el de la clínica móvil y es probable que la falta de adherencia al tratamiento terapéutico de dichos proyectos esté relacionada con el desconocimiento cultural de ambos constructos por parte del equipo profesional.

\section{REFERENCIAS BIBLIOGRÁFICAS}

1. Mikutu KA. Small arms and light weapons among pastoral groups in the Kenya-Uganda border área. African Affrairs. 2007;106(422):47-70.

2. Schelling E, Weibel D, Bonfoh B. Aprender de la provisión de servicios sociales a pastoralistas: elementos de una buena práctica [Internet]. Nairobi: PNUD; 2008 [citado 17 feb 2016]. Disponible en: https://goo.gl/9hsvHE.

3. Shankar D, Haverkort B. Vitalidad, salud y diversidad cultural. Boletín COMPAS. 2000;(3):4-7.

4. Terrence McCabe J. El impacto y la respuesta a la sequía entre los pastores turkanas: Implicaciones para la teoría antropológica y la investigación de riesgos. Desacatos. 2005;(19):25-40.
5. Hewitt K. The idea of calamity in a technocratic age. In: Hewitt K. Interpretations of Calamity. Winchester: Allen and Unwin; 1983. p. 3-32.

6. Glantz M. Drought and economic development in Sub-Saharan Africa. In: Glantz M. Drought and hunger in Africa: Denying famine a future. Cambridge: Cambridge University Press; 1987. p. 37-58.

7. García Acosta V. Historical disaster research. In: Hoffman SM, Oliver-Smith A, (eds.). Catastrophe \& culture: the anthropology of disaster. Santa Fe: School of American Research Press; 2002. p. 49-66.

8. Watts J, Hales H. A Turkana apron from Kenya [Internet]. 2011 [citado 19 may 2015]. Disponible en: http://goo.gl/DqsJjn. 
9. Sahlins MD. Las sociedades tribales. Barcelona: Labor; 1972.

10. Trip Down Memory Lane. Turkana people: Kenya's beautiful semi-nomadic Nilotic people [Internet]. 2013 [citado 14 jul 2015]. Disponible en: http://goo.gl/k3uUsM.

11. Abati FG. La religión de los Himba: Kaokoland (Namibia). Revista de Antropología Social. 1992;(1):151-166.

12. Schmidt W. Totemismus, viehzüchterischer Nomadismus und Mutterrecht. Anthropos. 191516;X-XI:593-643.

13. Bayardo SJV. Antología de textos clásicos de la psiquiatría latinoamericana. En: Bayardo SJV, Malpica CR, De Lara JG. Maestros de la psiquiatría mexicana. Guadalajara: Amaya Ediciones; 2011. p. 281-284

14. Fundación Emalaikat. Memoria de Actividades 2013 [Internet]. 2013 [citado 15 dic 2014]. Disponible en: http://goo.gl/Wo210p.

15. Good CM. Ethnomedical systems in Africa: Patterns of traditional medicine in rural and urban Kenya. New York: Guilford Press; 1987.

16. Gearhart R, Abdulrehman MS. Concepts of illness among the Swahili of Lamu, Kenya. Journal of Transcultural Nursing. 2014;25(3):218-222.

17. Swift J. Planning against drought and famine in Turkana, Northern Kenya. Nairobi: Oxfam, IDS, TRP; 1985.

18. Hendrickson D, Mearns R, Armon J. Livestock raiding among the pastoral Turkana of Kenya: redistribution, predation and the links to famine. Institute of Development Studies. 1996;27(3):17-30.

19. Wienpahl J. Livestock production and social organization among the Turkana (pastoralism, nomads; Kenya, East Africa) [Tesis de Doctorado en Internet]. Arizona: University of Arizona; 1984 [citado 15 ago 2015]. Disponible en: http://hdl. handle.net/10150/187667.

20. Figuera GDL. Mejora de las infraestructuras hidráulicas para la Comunidad Misionera de San Pablo Apóstol en Lobur, Turkana North District, Kenia [Disertación de Grado en Internet]. Madrid: Universidad Politécnica de Madrid; 2010 [citado 16 feb 2016]. Disponible en: http://oa.upm.es/5389/.

21. Villaseñor Bayardo SJ, Rojas Malpica C, Berganza Champagnac CE. La enfermedad y la medicina en las culturas precolombinas de América: la cosmovisión nahua. Investigación en Salud
[Internet]. 2002;IV(3) [citado 16 feb 2016]. Disponible en: http://goo.gl/3oiJT5.

22. Erkoreka A. Mal de ojo: una creencia supersticiosa remota, compleja, y aún viva. Munibe (Antropología-Arkeología). 2005;(57):392-400.

23. King B. Taking health care to the Maasai. Africa Health. 1992;14(2):27-30.

24. Cohen D. Providing nomadic people with health care. BMJ. 2005;331(7519):720.

25. Harragin S. Health and healthcare provision in North West Turkana, Kenya [Internet]. ODI Pastoral Development Network; 1994 [citado 22 feb 2016]. Disponible en: http://goo.gl/uyUXgL.

26. Sonkyo Energy. Instalación de un sistema híbrido para generación de electricidad: Turkana, Kenia [Internet]. Santander: Fundación ROSE; 2011 [citado 14 may 2015]. Disponible en: http:// goo.gl/nU1f7W.

27. Mbindyo $\mathrm{P}$, Blaauw D, English $M$. The role of clinical officers in the Kenyan health system: a question of perspective. Human Resources for Health. 2013;11(1):32.

28. Murillo J, Martínez C. Investigación etnográfica [Internet]. Madrid: Universidad Autónoma de Madrid; 2010 [citado 13 jul 2015]. Disponible en: http://goo.gl/AFdFf9.

29. Juma RO. Turkana livelihood strategies and adaptation to drought in Kenya. [Tesis de Doctorado en Internet]. New Zealand: Victoria University of Wellington; 2009 [citado 12 feb 2016]. Disponible en: http://hdl.handle.net/10063/1063.

30. Sundal MB. Difficult decisions: Karimojong healing in conflict [Tesis de Doctorado en Internet]. Kansas: University of Kansas; 2009 [citado 1 dic 2014]. Disponible en: http://hdl.handle. net/1808/6000.

31. Barrett AJ. Sacrifice and prophecy in Turkana cosmology. Nairobi: Paulines Publications Africa; 1998.

32. Sandford S. Welfare and wanderers: the organization of social services for pastoralists. Development Policy Review. 1978(A11);1:70-87.

33. Swift J, Toulmin C, Chatting S. Providing services to nomadic people: A review of the literature and annotated bibliography (Unicef Staff Working Papers 8). New York: Unicef; 1990.

34. Lillo-Crespo M. Asesoramiento en cuidados culturalmente competentes y evaluación de la 
satisfacción en el paciente holandés del plan de choque intereuropeo: una investigación cualitativa y transcultural en el contexto de la práctica de enfermería [Tesis de Doctorado en Internet]. Alicante: Universidad de Alicante; 2005 [citado 4 mar 2016]. Disponible en: http://hdl.handle. net/10045/10934.

35. Lillo-Crespo M, Vizcaya F, Domínguez JM, Galao R. Investigación cualitativa en enfermería y competencia cultural. Index de Enfermería. 2004;13(44-45):47-50.
36. Lillo-Crespo M, Sierras C, Cabañero MJ, Riquelme J. ISTEW European Project: Improvement Science Training for European Healthcare WorkersSpanish Contribution [Internet]. Global Science and Technology Forum: Annual Worldwide Nursing Conference 2015 doi: 10.5176/2315-4330_ WNC15.131.

\section{FORMA DE CITAR}

Cardós García JJ, Lillo Crespo M, Climent Rubio A, Hernández Urrutia E, Mejias Moreno B. Aproximación etnográfica al concepto de salud y enfermedad en la tribu turkana: proyecto para la mejora de la salud de los nómadas del Triángulo de Ilemi, Kenia. Salud Colectiva. 2016;12(2):173-188. doi: 10.18294/sc.2016.787

Recibido: 3 de noviembre de 2015 | Versión final: 14 de marzo de 2016 | Aprobado: 25 de abril de 2016

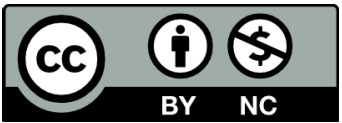

Este obra está bajo una licencia de Creative Commons Reconocimiento-NoComercial 4.0 Internacional. Reconocimiento - Permite copiar, distribuir y comunicar públicamente la obra. A cambio, se debe reconocer y citar al autor original. No Comercial - Esta obra no puede ser utilizada con finalidades comerciales, a menos que se obtenga el permiso. 